\title{
Predicting response to leuprolide of women with premenstrual dysphoric disorder by daily mood rating dynamics
}

\author{
Steven M. Pincus ${ }^{a},{ }^{,}$, Shaista Alam ${ }^{b}$, David R. Rubinow ${ }^{c}$, Chaya G. Bhuvanesward, and Peter \\ J. Schmidt ${ }^{b}$ \\ a990 Moose Hill Road, Guilford, CT 06437, USA \\ ${ }^{b}$ Behavioral Endocrinology Branch, National Institute of Mental Health, Bethesda, MD, USA \\ 'Department of Psychiatry, University of North Carolina School of Medicine, Chapel Hill, NC, USA \\ dDepartment of Psychiatry, University of Pennsylvania School of Medicine, Philadelphia, PA, USA
}

\begin{abstract}
Approximately 60-70 percent of women with premenstrual dysphoric disorder (PMDD) show symptomatic improvement in response to the GnRH agonist leuprolide acetate, which suppresses ovarian function. However, it has been very difficult to either predict or understand why some women respond, while others do not. We applied several complementary statistical methods to the dynamics of pre-treatment mood rating data to determine possible predictors of response for women with PMDD. We compared responders $(n=33)$ to nonresponders $(n=12)$ in clinical trials of leuprolide (three months in duration) as a treatment for PMDD, on the basis of pre-trial daily self-ratings of sadness, anxiety, and irritability. We analyzed both sequential irregularity (approximate entropy, ApEn) and a quantification of spikiness of these series, as well as a composite measure that equally weighted these two statistics. Both ApEn and Spikiness were significantly smaller for responders than nonresponders $(P \leq 0.005)$; the composite measure was smaller for responders compared with nonresponders $(P \leq 0.002)$ and discriminated between the subgroups with high sensitivity and specificity. In contrast, mean symptom levels were indistinct between the subgroups. Relatively regular and non-spiky pre-trial dynamics of mood ratings predict a positive response to leuprolide by women with PMDD with high probability, moreover based on typically less than 3 months of daily records. The statistical measures may have broad and direct applicability to behavioral studies for many psychiatric disorders, facilitating both accurate diagnosis and the prediction of response to treatment.
\end{abstract}

\section{Keywords}

Mood disorders; Premenstrual dysphoric disorder; Response to therapy; Approximate entropy; Cyclicity

\footnotetext{
(C) 2010 Elsevier Ltd. All rights reserved.

*Corresponding author. Tel.: +1 203 458 3455; fax: +1 203453 8969. stevepincus@ alum.mit.edu (S.M. Pincus).

Contributors

Steven Pincus contributed to study design, data analysis and interpretation, figure creation and manuscript preparation. Shaista Alam contributed to data analysis and interpretation and to manuscript preparation. David Rubinow contributed to data analysis and interpretation and to manuscript preparation. Chaya Bhuvaneswar initiated the study, and contributed to study design and manuscript preparation. Peter Schmidt managed all patients participating in the study, and contributed to study design, data analysis and interpretation and manuscript preparation.

Conflicts of interest statement

None declared.
} 


\section{Introduction}

During the past 20 years, premenstrual syndrome (PMS) has been the subject of extensive study. A severe form of PMS, premenstrual dysphoric disorder (PMDD), is a cyclical disorder characterized by mood-related and somatic symptoms that occur during the luteal phase of the menstrual cycle, disappear at or soon after the onset of menstruation, and are associated with significant impairment of function. Approximately 3-8\% of women experience PMDD, which has been estimated as resulting in 14.5 million disability adjusted life years annually in the United States (Halbreich et al., 2003; Johnson, 1987; Sternfeld et al., 2002). The precise etiology underlying PMDD is presently unknown. However, physical and behavioral symptoms are alleviated by suppressing ovarian activity (Brown et al., 1994; Hahn et al., 1995) and can be provoked in medically ovariectomized women by exposing them to exogenous gonadal steroids (Schmidt et al., 1998). Indeed, previous studies suggest that 60-70 percent of women with PMDD respond to the GnRH agonist leuprolide acetate (leuprolide) (Freeman et al., 1997; Mezrow et al., 1994; Mortola et al., 1991; Schmidt et al., 1998). It has been, however, a major puzzle as to why some women respond, while others do not.

The analysis of daily symptom ratings is a major component in the evaluation of PMS and PMDD. Specifically, to certify PMDD, there needs to be a substantial (oftentimes 30\%) increase in mean levels of negative moods in the week before menses compared to the week after the end of menses (American Psychiatric Association, 1994; Smith et al., 2003). Yet for many PMDD women, there often appears to be a clearly defined cyclical regularity in monthly ratings, beyond the requisite increase in mean negative emotions during the luteal phase. However, apart from the mean of selected time-periods, other serial characteristics of these mood rating sequences (time-series) and associated statistical measures generally remain unevaluated.

In a previous study of mood rating time-series characteristics of PMDD and recurrent brief depressive disorder (RBD) subjects, compared with each other and with normal controls, we identified two primary distinct, complementary statistical attributes that allowed us to statistically define each subgroup with high sensitivity and specificity, despite no differences in mean mood levels among the subgroups (Pincus et al., 2008). The first attribute is sequential irregularity (approximate entropy, ApEn), which identifies the existence and degree of order and regularity ('extent of pure cyclicity') in what otherwise may be viewed as random or disordered serial data. The second attribute, motivated by what appears to be the defining characteristic of RBD, is the extent of brief sharp increases and decreases in mood levels, quantified by a measure denoted 'Spikiness'. As noted below, ApEn has been widely applied throughout biology and medicine, including many studies of hormonal dynamics of comparable data length and 'noise' (measurement inaccuracy) to those encountered in mood rating studies. More recently, ApEn has also been applied to a mood rating study contrasting three treatment regimens applied to healthy controls, with very significant decrease in mood dynamic irregularity seen for one of the treatments, among the regimens, despite no changes in mean levels (Yeragani et al., 2003), suggesting the utility of ApEn to quantify relatively subtle changes in mood during therapy. Consequently, we asked whether evaluation of pre-trial mood rating dynamics by ApEn and Spikiness in women with PMDD who undertook a subsequent trial of leuprolide would provide enhanced prediction of successful response to the therapy.

In the aforementioned study of mood rating dynamics of PMDD and RBD, we determined that compared with the other subgroups, PMDD subjects exhibited less irregularity and Spikiness, i.e., were smoother, more purely cyclical, and with fewer abrupt changes upwards or downwards in their moods. Notably, as considered further in the DISCUSSION, prior 
studies (Freeman et al.,1997, 2004) have shown that womenwith PMDD or severe PMS showed a poorer response to therapy if they exhibited mood dynamics that in the present context equate to either several instances of significant off-menses spikes or substantial deviations from cyclicity. Accordingly, the purpose of this study was to evaluate the resultant hypothesis that smaller ApEn (greater regularity) and lower Spikiness of mood ratings would predict successful response to leuprolide, while higher values of either or both measures would predict unsuccessful response, concomitantly segmenting women with PMDD into phenotypes based on their mood rating dynamics.

\section{Materials and methods}

\subsection{Subject selection}

Women with PMDD were self-referred in response to advertisements for women with distressing premenstrual mood symptoms in local newspapers or referred by their physician. All women were without current medical illness (as assessed by medical history, physical examination, laboratory tests, and electrocardiogram) and medication free. PMDD was prospectively confirmed by longitudinal ratings for three consecutive cycles. Before entry into the study, prospective participants were screened with a daily visual-analogue scale (VAS) of self-ratings of mood. All PMDD patients experienced at least 5 of the DSM-IV symptoms for PMDD (American Psychiatric Association,1994) associated with significant functional impairment; additionally, each PMDD patient in the present study showed at least a $30 \%$ increase in mean levels (relative to the range of the scale employed) of negative moods (sadness, anxiety, and/or irritability) in the week before menses compared with the week after the end of menses in at least two of three menstrual cycles. Current and past histories of psychiatric disorders were obtained with the SCID (Structured Clinical Interview) for DSM III-R for women recruited prior to 1996, and with the SCID for DSMIV for those recruited during or after 1996 (Spitzer et al., 1990). PMDD subjects were not permitted to have either a current or recent past (last two years) history of any disorder other than PMDD.

Subjects for the current study were selected from patients seen in our clinic. Of the 274 women who met the aforementioned diagnostic criteria for PMDD between 1992 and 2008, 45 women were admitted to a trial of depot leuprolide as a treatment of PMDD, in either a double blind placebo-controlled trial $(n=10)$ or an open label trial $(n=35)$. Thirty three women (age $=38.8 \pm 6.5$ years) met our criteria for response, defined below, and twelve women $(\mathrm{age}=40.8 \pm 5.3$ years $)$ did not meet the criteria.

All reported regular menstrual cycles (range, 21-35 days), and all had a negative urine pregnancy test, normal thyroid levels, and a normal physical examination, including Pap smear. None were on any hormonal based birth control at intake or at time of testing or within the previous year. The protocols were reviewed and approved by the National Institute of Mental Health (NIMH) institutional review board, and all subjects gave both written and verbal consent to study participation.

Upon completion of screening and preceding the start of leuprolide, daily self-rating mood data were recorded for 2-4 months. We utilized a 3-item visual-analogue scale (VAS) to assess the severity of common symptoms of PMDD (Rubinow et al., 1984; Schnurr, 1988, 1989). The VAS forms were completed once daily (evening) during the three-month baseline phase; the women were instructed to rate how they felt at the moment they were completing the form. Herein, we analyze each of the sadness, anxiety, and irritability symptom data sets as time-series. For each time-series, we evaluate the mean, irregularity (ApEn), and extent of staccato variation ('Spikiness' statistic), the last two of which are described below. In addition to the VAS, the women were asked to do Premenstrual Tension 
Syndrome Self Rating Scale (PMTS) cross-sectional ratings. In the 10-item scale used, a rater assesses the severity of common symptoms of premenstrual syndrome on a 4-point scale (with the exception of eating habits and sexual drive, which are evaluated on a 2-point scale); a score of 0 indicates the absence of symptoms, and a score of 36 indicates that all symptoms are present and severe (Steiner et al., 1980).

\subsection{Leuprolide trial}

The women in leuprolide trials made biweekly clinical visits to the NIH. The trial involved injections of depot leuprolide acetate $(3.75 \mathrm{mg})$ between days 2 and 6 after the onset of menses for 3 months to evaluate the response of medically induced ovarian suppression. Subjects who responded to leuprolide were continued for a second study phase during which they received the GnRH agonist for an additional 3 months and were replaced with physiological levels of estradiol and progesterone. For the purposes of this paper, we are limiting the study to the effects of leuprolide alone. Further details can be found elsewhere (Schmidt et al., 1998).

\subsection{Criteria for response}

Response to leuprolide was defined by both (i) the absence of significant symptom cyclicity for each of sadness, anxiety, or irritability, and (ii) the absence of PMTS ratings $>10$ during months 2-3 on leuprolide. To specify (i), significant symptom cyclicity was defined by a symptom VAS score greater than $30 \%$ below the overall mean (during the symptomatic baseline) which persisted for at least 2 consecutive days during months 2 and 3 of leuprolide treatment. For (ii), symptoms were assessed by the 10-item PMTS ratings.

\subsection{Hormonal, hot flush data}

To assess further potential contributors to response, we measured hormonal and hot flush data. For the former, blood samples were drawn every two weeks throughout the study. The samples were centrifuged, and aliquots of plasma were frozen at $-20^{\circ} \mathrm{C}$ until the time of assay (Schmidt et al., 1998). Plasma progesterone, estradiol, luteinizing hormone (LH) and follicle stimulating hormone (FSH) were measured by radioimmunoassay as previously described (Schmidt et al., 1998). For the latter, the women recorded the presence and severity of hot flushes (a potential side effect of leuprolide) daily, employing a 6-point likert-type scale (see Table 1).

Finally, clinical reports of the exacerbation of PMDD during the four weeks after leuprolide administration (when administered in the early follicular phase) suggest that the initial hormonal flare triggers symptoms in these women and could predict elimination of symptoms when ovarian steroids are suppressed. Thus, we examined the relationship between symptom exaggeration during the first month of leuprolide (i.e., symptom flare) and response to leuprolide. A symptom flare was defined by the threshold values of anxiety, irritability and sadness from the baseline mood ratings of patients before they started leuprolide. The threshold value for each symptom was defined as the value obtained by subtracting $30 \%$ of the range of VAS from the baseline mean of the particular symptom. A flare was confirmed if any mood ratings exceeded the threshold values for two or more consecutive days during the first month of leuprolide.

\subsection{Analytic strategy}

2.5.1. Quantification of irregularity-To quantify sequential irregularity, we utilize approximate entropy, ApEn, a model-independent measure defined by Pincus (1991). Primary mathematical and statistical properties of ApEn, including error estimation for general processes and robustness to noise and artifacts can be found elsewhere (Pincus and 
Goldberger, 1994; Pincus and Huang, 1992; Pincus and Kalman, 1997; Pincus and Singer, 1996). ApEn was introduced to quantify regularity in sequences and time-series data, initially motivated by applications to relatively short, noisy data (Pincus, 1991). It has been used extensively to characterize the degree of randomness in a variety of applications that explore regularity in physiological systems including patterns of hormonal secretion (Pincus et al., 1998, 1999), heart rate (Pincus et al., 1993), respiration (Akay et al., 2002; Yeragani et al., 2002) and EEG dynamics (Bruhn et al., 2000). Additionally, ApEn provides a direct barometer of feedback system change in many coupled systems (Pincus, 1994; Pincus and Keefe, 1992).

ApEn calibrates an extent of serial interrelationships, quantifying a continuum that ranges from totally ordered to completely random. ApEn assigns a nonnegative number to a timeseries, with larger values corresponding to greater apparent process randomness (serial irregularity), and smaller values corresponding to more instances of recognizable features or patterns in the data. Two input parameters, $m$ and $r$, must be specified to compute ApEn. Briefly, ApEn measures the logarithmic likelihood that runs of patterns that are close (within $r$ ) for $\mathrm{m}$ contiguous observations remain close (within the same tolerance width $r$ ) on next incremental comparisons; the precise mathematical definition is given in Pincus (1991).

We calculated ApEn values for each time-series with input parameters $m=1$ and $r=20 \%$ of the standard deviation (SD) of the individual subject time-series, the standard choice for this data length (Pincus and Goldberger, 1994; Pincus et al., 1999). Normalizing $r$ to each timeseries SD gives ApEn a translation- and scale-invariance to absolute levels, in that it remains unchanged under uniform process magnification, reduction, or constant shift higher or lower (Pincus et al., 1993). Multiple previous studies that included both theoretical analysis and clinical applications (Pincus, 1991; Pincus et al., 1999) have demonstrated that the input parameters indicated above produce good statistical validity (reproducibility) for ApEn for time-series of the length considered herein. Since the ApEn expected value increases slightly with increasing series length, we control for this by analyzing (and reporting) ApEn values calculated as a fraction of the maximal possible ApEn value for the given data length (Veldhuis et al., 2008).

Below, ApEn is applied to both the raw data and to logarithmically transformed time-series, as it was applied in our previous study (Pincus et al., 2008). The latter analysis, widely used in many application areas, is complementary to the analysis of the raw data, as it generally lessens the effects of steep local descents or ascents, of outliers, and of heteroscedasticity (changing variance), while still preserving the relative order of the data.

2.5.2. Spikiness-A relatively subtle yet important difference between patient groups can sometimes be seen by the presence or absence of acute sharp increases and decreases in mood levels. We quantify the extent of such sharp changes by Spikiness, defined as the ratio of the SD of the first differenced (incremental process) series and the SD of the original series of mood ratings. Descriptively, Spikiness assesses the absolute magnitude of incremental change or day-today variation of the original series, normalized (relative) to overall variation. As discussed in Pincus et al. (2008), this formulation is both robust and broadly applicable to general time-series, and to either linear or non-linear models.

This normalization to SD is consistent with other model-dependent formulations of steepness of ascent near a peak such as kurtosis (Press et al., 1986) and crest factor (Schoukens and Pintelon, 1991). Our preference for the specification of Spikiness, compared to these thematically similar formulations, is dictated by several factors. First and primary, both of these alternatives are intended for single peak or unimodal distributions, rather than multiple peak or more general settings, and therefore are more limited in scope. 
Additionally, kurtosis is not always robust for shorter data sets, as it requires a fourth moment estimate, while crest factor requires clear peak identification.

Mathematically, Spikiness and ApEn are defined to assess separate facets of dynamical behavior in time-series. Generally, highly irregular data sets also tend to have high Spikiness, but otherwise, both highly regular (low ApEn) and moderately irregular timeseries can exhibit either high or low Spikiness. This distinction between Spikiness and ApEn is empirically seen in a previous study (Fig. 4 in Pincus et al., 2008).

The clinical utility of Spikiness is also seen in Pincus et al. (2008), in which patients with recurrent brief depression had relatively large values of Spikiness, manifesting sharp changes in state that underlie the brevity of the depression. This significantly contrasted with lower values of Spikiness for women with PMDD, for whom the day-to-day mood variation (hence the numerator in the Spikiness definition) is small relative to the overall SD for patients in this subgroup.

2.5.3. Index of smooth cyclicity-Upon the quantifications by ApEn and Spikiness, we have two complementary sets of statistics to assess deviations from purely cyclic curves (e.g., sine waves). Since high values of either measure violate our perception of cyclicity, we aimed to combine these two measures into a single, statistically appropriate index that quantifies deviations from either regularity or smoothness. Accordingly, we combined ApEn and Spikiness into a measure, denoted the Index of smooth cyclicity, by averaging equally weighted values of ApEn and Spikiness (i.e., standardized to overall population ApEn and Spikiness SDs). Irregular and spiky dynamics produce high index values, whereas regular mood rating profiles with few large spikes produce low index values. We formed two such indices, one based on ApEn calculated from the log-transformed series, the other from ApEn calculated on the original series. Based on the previous study in which we applied ApEn to both original and log-transformed data, we anticipated that the log-transformed version would provide slightly better discrimination between subgroups, and thus might be more likely to be applied in subsequent studies. Hence, and to distinguish between versions of this index, below we denote the Index of smooth cyclicity as the averaged version incorporating the log-transformed ApEn value, and refer to the other composite measure as the normalized average of ApEn and Spikiness.

\subsection{Statistical analysis}

For each participant and statistic under study, in addition to calculating values for each symptom, we also calculated the average value of the three individual symptom scores. Notably, a low averaged value of the Index of smooth cyclicity typically manifests consistency in both regularity and non-Spikiness across the three symptoms, throughout the mood rating study period.

Statistical comparisons for discrimination between responder and nonresponder subgroups (including demographic, hormonal and flare characteristics) were performed with both (i) the two-sided $t$-test with unknown variance, and (ii) the nonparametric rank-sum test for numerical data, and (iii) the chi-square test for categorical data. The application of both parametric and nonparametric statistical techniques was chosen to insure robustness of the results to a range of distributional assumptions. In particular, the individual subject distributions for ApEn, Spikiness and the Index of smooth cyclicity appear to differ considerably from a normal distribution (Fig. 1). Odds ratios were also calculated for specified threshold levels. All findings were deemed significant at a 95\% confidence level. 


\section{Results}

None of the demographic or baseline characteristics were significantly different between the responders and the nonresponders (Table 1). Specifically, there were no significant differences between responders and nonresponders in the percentage of women who experienced a clinically significant symptom exacerbation (flare) after the first injection of leuprolide. Also, nonresponders did not report significantly more severe hot flushes than responders, and measures of ovarian suppression, including plasma ovarian steroid levels and gonadotropin levels, did not significantly differ between responders and nonresponders. Thus, ovarian function in nonresponders was equally suppressed as that in responders, albeit plasma gonadotropin levels, in particular, plasma LH, were non-significantly higher in the nonresponders compared with the responders. Finally, although nonresponders had slightly higher rates of a past history of major depression than did the responders, this difference did not reach statistical significance.

Representative pre-trial daily mood rating time-series for two responders and two nonresponders are shown in Fig. 2. For the responders, observe the 'classic' cyclic dynamics for each symptom, whereas for the nonresponders, observe the inconsistency in mood dynamics from month to month, and numerous brief moderate-to-large drops in mood, or depressive epochs, not proximate to menses.

Table 2 indicates the mean \pm SD for each of the six statistics under study (mean, Spikiness, ApEn, ApEn of the log-transformed series, Index of smooth cyclicity, and normalized average of ApEn and Spikiness) by subgroup, both averaged across the three VAS symptoms (A) and for each individual symptom (B-D). Furthermore, Table 2 indicates the sensitivity and specificity of each measure, with associated cutpoint (threshold), calculated from receiver operating characteristic (ROC) curve analysis to maximize sensitivity plus specificity. Individual subject values for each of these statistics by subgroup are displayed in Fig. 1, for the data summarized in Table 2A.

There were no significant differences in mean levels between the responders and nonresponders, $P>0.25$ for both $t$-test and rank-sum test, for the aggregate (average of three symptoms) measure and for each individual symptom. Each of the other measures were significantly smaller for responders than nonresponders for virtually all other comparisons, most pointedly realized for the comparisons based on averages of the individual (VAS) symptom calculations (Table 2A), for which all non-mean comparisons showed differences with significance $P \leq 0.006$. The greatest significance was seen for the Index of smooth cyclicity, $P=0.0012$, with the cohort difference visually confirmed by Fig. 1(F).

For the Index of smooth cyclicity (for the averaged VAS symptom calculations), we also calculated odds ratios for two distinct thresholds, one higher and one lower. For the higher threshold, 7 of 12 nonresponders (58.3\%) and 1 of 33 responders (3.0\%) had index values $\geq 0.72$ (Odds ratio, $44.8 ; 95 \% \mathrm{CI}, 4.50$ to $445.75 ; P=0.001$ ). For the lower threshold, 23 of 33 responders $(69.7 \%)$ and 2 of 12 nonresponders $(16.7 \%)$ had index values $\leq 0.62$ (Odds ratio, $11.5 ; 95 \% \mathrm{CI}, 2.12$ to $62.32 ; P=0.004)$.

Finally, there were no significant correlations between mean levels and any of the other five measures of temporal dynamics under study.

\section{Discussion}

The present analysis compares responders with nonresponders in leuprolide treatment of PMDD, in a study cohort that includes, yet enlarges, the group of women evaluated in a previous study (Schmidt et al., 1998). Seventy-three percent (33/45) of women responded to 
therapy, showing decrease of symptom severity and elimination of symptom cyclicity. This response rate is slightly but insignificantly larger than that seen in previous studies (Freeman et al., 1997; Mezrow et al., 1994; Mortola et al., 1991; Schmidt et al., 1998), and reaffirms the efficacy of GnRH agonists in a majority of, but not all, women with PMDD.

\subsection{Subgroup differences}

The primary objective of the present study was to determine possible predictors of response to leuprolide, based on VAS mood rating records obtained prior to onset of therapy. Quantification of each of serial irregularity (ApEn) and the extent of brief, staccato dynamics (Spikiness) in pre-trial mood ratings records enabled us to significantly discriminate between responders and nonresponders. Furthermore, when the two complementary statistics were combined into a single Index of smooth cyclicity, and averaged across symptoms, an even more notable distinction emerged between the two cohorts.

Very high index values were highly specific to nonresponse; similarly, very low index values were very specific to response, quantified by the odds ratio calculations. Descriptively, those subjects who were most consistently highly regular or cyclic in their mood dynamics, across three symptoms and several months, and who also had few brief spiky periods (negative or positive), showed responsiveness to leuprolide, while those subjects at the other end of the statistical spectrum here responded poorly. The quantification of a consistency of 'purely cyclic' symptomatology across the three axes formalizes yet strengthens the clinician's intuitive diagnosis of PMDD. Notably, we show that such consistency, appropriately quantified, defines a phenotype that predicts very high likelihood of therapeutic success with leuprolide.

There were no significant differences in mean sadness levels between responders and nonresponders. This reinforces the utility of looking beyond the mean mood level to assess affective state. This is not surprising - PMDD is an affective disorder defined by its cyclicity as much as by associated symptoms. Hence, attempts to characterize this disorder on the basis of mean or peak symptom levels fail to consider mood regulatory dynamics that are essential to PMDD, and prompt the application of alternative statistical methods to suitably quantify disrupted dynamics.

Although statistical comparisons disclosed significant differences between responders and nonresponders for each of the three negative emotions, we realized clearest discrimination upon averaging each of ApEn and Spikiness across the three symptoms (Table 2). This is not unexpected. Although the DSM-IV mandates the presence of at least one (of four) core symptoms, it does not specify the particular symptom(s), which often vary across women, sometimes even across months for individuals. Thus our aggregate, averaged measures ensure coverage of at least one symptom for all women, whereas no pre-specified mood analyzed necessarily need exhibit substantial cyclic monthly variation.

There is a natural biologic interpretability to the relatively small ApEn values seen for the PMDD subgroup. Clinically, the observation of premenstrual sadness followed by postmenstrual euphoria provides a predictability or regularity to monthly dynamics, commensurate with lower ApEn values, in contrast to a more stable baseline for normal women, for whom mood variations are less tied to any specific fixed interval, appearing less predictable, manifested by larger ApEn values (Pincus et al., 2008).

\subsection{Clinical implications}

Several methods have been developed to confirm the diagnosis of women with PMDD and/ or PMS (Angst et al., 2001; Freeman and Halbreich, 1998; Freeman et al., 1985; Yonkers et 
al., 1997). A protracted debate has accompanied the specification of diagnostic criteria, as many women meeting criteria for severe PMS also meet criteria for PMDD. In brief, the American Psychiatric Association espouses classification of PMDD as an affective disorder, with primary symptoms the mood problems - uniform diagnostic criteria are listed in DSMIV (American Psychiatric Association, 1994). In contrast, the American College of Obstetricians and Gynecologists provides diagnostic criteria for PMS (American College of Obstetricians and Gynecologists, 2000), with greater emphasis on prominent somatic symptoms. Both PMS and PMDD are defined largely, albeit not exclusively, on the basis of mean level changes in specified attribute measures. Accordingly, the methods and results reported herein may prove useful in specifying quantitative characteristics of mood and physical dynamics of the disorder, either towards a single, unified severe form entity, or to multiple distinct phenotypes that provide more precise syndromal classification for the evaluation of treatment response. Above, we already realize a reduction in the heterogeneity of treatment effects, an important concern in randomized controlled trials (Kent and Hayward, 2007; Kravitz et al., 2004), upon restriction to phenotypes solely defined by either very low or very high index values. Further fundamental understanding and potential utility could be derived from associations between statistically defined phenotypes and (i) dysregulation in concomitant physiological variables, and (ii) specific genotypes.

It is well known that symptom characterization and severity across menstrual cycles in women with PMS are inconsistent (Schnurr,1988; Smith et al., 2003). In many studies of PMS and PMDD, analyses are restricted to symptomatic cycles, which helps to decrease cross-study variability (Angst et al., 2001; Smith et al., 2003). However, firm criteria to certify individual cycles as symptomatic or asymptomatic are not definitive (Smith et al., 2003), in part manifesting the lack of complete overlap between PMS and PMDD specifications. A direct application of ApEn to an entire mood study record of at least several months obviates an a priori need to assess or identify individual cycles as symptomatic. Instead, such a direct application in effect assesses the degree of consistency of symptomatic vs. asymptomatic cycles. Intermittently symptomatic women will generally register higher ApEn scores than will consistently symptomatic women, marking the greater mood irregularity. Indeed, one primary interpretation of the results above is that greater consistency in symptomatic months in part defines a phenotype that is more likely to exhibit positive response to leuprolide (and quite possibly to other similar medications). Moreover, it is remarkable that we achieve the level of significance in comparisons herein based on only $2-3$ months of data per subject, although the simultaneous analysis of multiple symptoms likely abets our present analyses. We anticipate that longer (e.g., 6 month) mood rating studies, although somewhat less feasible, would provide yet finer definition of the degree of monthly consistency of symptomatology and further precision in identifying potential responders.

It is both striking and of considerable practical import that the statistically defined distinctions were accomplished without either any incorporation of dates of menses or a protocol mandate of a common start date with respect to menses. In effect, one of our primary study conclusions is that for subjects with a low value of the Index of smooth cyclicity, the native mood cyclicity itself is sufficiently well-defined that quantification of its degree does not depend on cycle start-point, so long as two or more months of accurately reported data are available.

The present findings reinforce the utility of daily recording of mood ratings during a study period, since it is in analyses beyond mean level comparisons that we deduce the distinctions seen here. In the absence of relatively accurate records, estimates of measures such as ApEn, Spikiness, or other dynamic features would be considerably compromised, and accordingly, so would the possibility of identifying proper phenotyping on the basis of these measures. 
Previous studies have disclosed a 60-70\% efficacy rate of therapy for PMDD for GnRH agonists (Freeman et al., 1997; Mezrow et al., 1994; Mortola et al., 1991; Schmidt et al., 1998), and a 48-63\% rate for selective serotonin reuptake inhibitors (SSRIs) (Freeman et al., 2004) and for a low dose oral contraceptive pill (OCP) formulation containing drospirenone $3 \mathrm{mg}$ and ethinyl estradiol $20 \mu \mathrm{g}$ (Yonkers et al., 2005). Nonetheless, and importantly, despite very distinct pharmacological strategies, in comparison studies, the rate of treatment response has barely exceeded $20 \%$ above placebo (Halbreich, 2004), suggesting that only a subgroup of women with PMDD respond to these medications. One primary potential application of the present findings is the evaluation of phenotype-targeted treatments. We hypothesize that the efficacy of the aforementioned (and possibly other) therapies will be considerably larger than $20 \%$ above placebo treatment, when restricted to studies of PMDD patients with a low Index of smooth cyclicity, i.e., those with regular and non-spiky dynamics.

Further support for this hypothesis comes from reevaluation of previous studies in the present context. Freeman et al also showed that patients either with a past history of depression, or in a premenstrual exacerbation group with dysphoric symptoms throughout the cycle, had a much lower response rate to leuprolide therapy, compared to a clearly defined PMS subgroup (Freeman et al., 1997). Additionally, women with PMDD or severe PMS who exhibited higher postmenstrual symptom levels ("a possible indication of subclinical levels of depression") showed a poorer response to sertraline treatment (Freeman et al., 2004). In effect, co-mingled instances of acycylic depressive episodes or subclinical dysphoric tendencies are predictors of poorer response, and a low Index of smooth cyclicity implies that there are few instances of either 'risk factor', as assessed along two distinct statistical axes. Thus many of the women in the aforementioned two studies with a reduced likelihood of successful therapy would generally be excluded from a low Index of smooth cyclicity cohort, on the basis of firm quantitative criteria. Finally, we remark that the sertraline study (Freeman et al., 2004) utilized an SSRI, not a GnRH agonist, providing further support that the identification of smoothly cyclic women with PMDD has potential utility in the evaluation of a range of therapies across multiple pharmacological categories.

Finally, the negative findings in the comparisons of hormone levels are not surprising. It has been well established that in many instances in which mean levels are unchanged, disruptions in pulsatility patterns of hormonal dynamics provide primary characterization of many (endocrinologic) pathologies, in part motivating the extensive application of ApEn to this area of research (Pincus et al., 1996, 1998, 1999). Patients with PMS have previously demonstrated altered secretory patterns of progesterone (Facchinetti et al., 1993) and LH (Facchinetti et al., 1990). Notably, in a recent study of hypothalamic-pituitary-adrenal (HPA) axis data in men with major depressive disorder (MDD), the depressed men had significantly altered cortisol and ACTH ApEn compared with healthy controls, while depressed patients and controls did not differ significantly on mean level indices of basal hormone secretion (the latter consistent with prior studies) (Posener et al., 2004). Similarly, despite little evidence of abnormal ovarian mean hormonal levels in PMS, there is substantial evidence that gonadal steroids nonetheless trigger or play an important role in the etiology of PMS (Muse et al., 1984; Rubinow et al., 1988; Schmidt et al., 1991).

\subsection{Perspective and conclusion}

Above, we have quantified two distinct, complementary statistical attributes of daily mood rating dynamics to differentiate responders from nonresponders to leuprolide therapy in women with PMDD, in the process providing enhanced phenotypic definition of this disorder. Our study protocol fits into an orientation to better utilize extant or readily obtainable data from women with PMDD, requiring a minimal burden and expense for the subjects, over a relatively short ( $2-3$ month) and typically quite feasible time course. The 
results here also validate the utility of relatively accurate record keeping of mood ratings on a daily basis. Furthermore, upon delineation of PMDD into phenotypes on the basis of regularity and Spikiness, we anticipate the possibility of considerably greater than the present $20 \%$ ceiling in therapeutic response rate (above placebo) in the subgroup with regular and non-spiky dynamics. Of course, future studies should be performed both to confirm the present findings, and particularly to further specify this response rate.

The results are directly and immediately applicable to the prediction of response to leuprolide therapy in women with PMDD. Finally, more broadly, the analytic methods employed, which can typically be applied as a 'black box' statistical suite, have considerable potential utility to studies of many mood disorders throughout psychiatry, either applied to the present VAS or other mood rating scales, or to the dynamics of any of several physiological signals.

\section{Acknowledgments}

We are indebted to Linda Schenkel for her expert data management in support of this study.

\section{Role of funding source}

This work was supported by the National Institute of Mental Health's Intramural Research Program. The NIMH had no further role in study design; in the collection, analysis and interpretation of data; and in the decision to submit the manuscript for publication.

\section{References}

Akay M, Lipping T, Moodie K, Hoopes PJ. Effects of hypoxia on the complexity of respiratory patterns during maturation. Early Human Development. 2002; 70:55-71. [PubMed: 12441205]

American College of Obstetricians and Gynecologists. ACOG practice bulletin 15. Washington, DC: ACOG; 2000. Clinical management guidelines for obstetricians-gynecologists:premenstrual syndrome.

American Psychiatric Association. Diagnostic and statistical manual of mental disorders. 4. Washington, DC: American Psychiatric Association; 1994.

Angst J, Sellaro R, Stolar M, Merikangas KR, Endicott J. The epidemiology of perimenstrual psychological symptoms. Acta Psychiatrica Scandinavica. 2001; 104:110-6. [PubMed: 11473504]

Brown CS, Ling FW, Andersen RN, Farmer RG, Arheart KL. Efficacy of depot leuprolide in premenstrual syndrome: effect of symptom severity and type in a controlled trial. Obstetrics and Gynecology. 1994; 84:779-86. [PubMed: 7936512]

Bruhn J, Ropcke H, Rehberg B, Bouillon T, Hoeft A. Electroencephalogram approximate entropy correctly classifies the occurrence of burst suppression pattern as increasing anesthetic drug effect. Anesthesiology. 2000; 93:981-5. [PubMed: 11020750]

Facchinetti F, Genazzani AD, Martignoni E, Fioroni L, Nappi G, Genazzani AR. Neuroendocrine changes in luteal function in patients with premenstrual syndrome. Journal of Clinical Endocrinology \& Metabolism. 1993; 76:1123-7. [PubMed: 8496301]

Facchinetti F, Genazzani AD, Martignoni E, Fioroni L, Sances G, Genazzani AR. Neuroendocrine correlates of premenstrual syndrome: changes in the pulsatile pattern of plasma LH. Psychoneuroendocrinology. 1990; 15:269-77. [PubMed: 1965049]

Freeman EW, Halbreich U. Premenstrual syndromes. Psychopharmacology Bulletin. 1998; 34:291-5. [PubMed: 9803757]

Freeman EW, Rickels K, Sondheimer SJ, Polansky M, Xiao S. Continuous or intermittent dosing with sertraline for patients with severe premenstrual syndrome or premenstrual dysphoric disorder. American Journal of Psychiatry. 2004; 161:343-51. [PubMed: 14754784]

Freeman EW, Sondheimer SJ, Rickels K. Gonadotropin-releasing hormone agonist in the treatment of premenstrual symptoms with and without ongoing dysphoria: a controlled study. Psychopharmacology Bulletin. 1997; 33:303-9. [PubMed: 9230648] 
Freeman EW, Sondheimer S, Weinbaum PJ, Rickels K. Evaluating premenstrual symptoms in medical practice. Obstetrics and Gynecology. 1985; 65:500-5. [PubMed: 4039044]

Hahn PM, Van Vugt DA, Reid RL. A randomized, placebocontrolled, crossover trial of danazol for the treatment of premenstrual syndrome. Psychoneuroendocrinology. 1995; 20:193-209. [PubMed: 7899538]

Halbreich U. The diagnosis of premenstrual syndromes and premenstrual dysphoric disorder - clinical procedures and research prespectives. Gynecological Endocrinology. 2004; 19:320-34. [PubMed: 15724807]

Halbreich U, Borenstein J, Pearlstein T, Kahn LS. The prevalence, impairment, impact, and burden of premenstrual dysphoric disorder (PMS/PMDD). Psychoneuroendocrinology. 2003; 28(Suppl 3):123.

Johnson SR. The epidemiology and social impact of premenstrual symptoms. Clinical Obstetrics and Gynecology. 1987; 30:367-76. [PubMed: 3301135]

Kent DM, Hayward RA. Limitations of applying summary results of clinical trials to individual patients: the need for risk stratification. Journal of the American Medical Association. 2007; 298:1209-12. [PubMed: 17848656]

Kravitz RL, Duan N, Braslow J. Evidence-based medicine, heterogeneity of treatment effects, and the trouble with averages. Milbank Quarterly. 2004; 82:661-87. [PubMed: 15595946]

Mezrow G, Shoupe D, Spicer D, Lobo R, Leung B, Pike M. Depot leuprolide acetate with estrogen and progestin add-back for long-term treatment of premenstrual syndrome. Fertility and Sterility. 1994; 62:932-7. [PubMed: 7926137]

Mortola JF, Girton L, Fischer U. Successful treatment of severe premenstrual syndrome by combined use of gonadotropin-releasing hormone agonist and estrogen/progestin. Journal of Clinical Endocrinology \& Metabolism. 1991; 72:252A-F.

Muse KN, Cetel NS, Futterman LA, Yen SSC. The premenstrual syndrome: effects of "medical ovariectomy”. New England Journal of Medicine. 1984; 311:1345-9. [PubMed: 6387488]

Pincus SM. Approximate entropy as a measure of system complexity. Proceedings of the National Academy of Science. 1991; 88:2297-301.

Pincus SM. Greater signal regularity may indicate increased system isolation. Mathematical Biosciences. 1994; 122:161-81. [PubMed: 7919665]

Pincus SM, Cummins TR, Haddad GG. Heart rate control in normal and aborted SIDS infants. American Journal of Physiology. 1993; 264:R638-46. [PubMed: 8457020]

Pincus SM, Goldberger AL. Physiological time-series analysis: what does regularity quantify? American Journal of Physiology. 1994; 266:H1643-56. [PubMed: 8184944]

Pincus SM, Hartman ML, Roelfsema F, Thorner MO, Veldhuis JD. Hormone pulsatility discrimination via coarse and short time-sampling. American Journal of Physiology. 1999; 277:E948-57. [PubMed: 10567024]

Pincus SM, Huang WM. Approximate entropy: Statistical properties and applications. Communications in Statistics - Theory and Methods. 1992; 21:3061-77.

Pincus S, Kalman RE. Not all (possibly) "random" sequences are created equal. Proceedings of the National Academy of Science. 1997; 94:3513-8.

Pincus SM, Keefe DL. Quantification of hormone pulsatility via an approximate entropy algorithm. American Journal of Physiology. 1992; 262:E741-54. [PubMed: 1590385]

Pincus SM, Mulligan T, Iranmanesh A, Gheorghiu S, Godschalk M, Veldhuis JD. Older males secrete luteinizing hormone $(\mathrm{LH})$ and testosterone more irregularly, and jointly more asynchronously, than younger males. Proceedings of the National Academy of Science. 1996; 93:14100-5.

Pincus SM, Padmanabhan V, Lemon W, Randolph J, Midgley AR. FSH is secreted more irregularly than LH in both human and sheep. Journal of Clinical Investigation. 1998; 101:1318-24. [PubMed: 9502773]

Pincus SM, Schmidt PJ, Palladino-Negro P, Rubinow DR. Differentiation of women with premenstrual dysphoric disorder, recurrent brief depression, and healthy controls by daily mood rating dynamics. Journal of Psychiatric Research. 2008; 42:337-47. [PubMed: 17336329]

Pincus S, Singer BH. Randomness and degrees of irregularity. Proceedings of the National Academy of Science. 1996; 93:2083-8. 
Posener JA, DeBattista C, Veldhuis JD, Province MA, Williams GH, Schatzberg AF. Process irregularity of cortisol and adrenocorticotropin secretion in men with major depressive disorder. Psychoneuroendocrinology. 2004; 29:1129-37. [PubMed: 15219636]

Press, WH.; Flannery, BP.; Teukolsky, SA.; Vetterling, WT. Numerical recipes: the art of scientific computing. New York: Cambridge University Press; 1986. p. 457-9.

Rubinow DR, Hoban MC, Grover GN, Galloway DS, Roy-Byrne P, Andersen R, et al. Changes in plasma hormones across the menstrual cycle in patients with menstrually related mood disorder and in control subjects. American Journal of Obstetrics and Gynecology. 1988; 158:5-11. [PubMed: 2962499]

Rubinow DR, Roy-Byrne PP, Hoban MC, Gold PW, Post RM. Prospective assessment of menstrually related mood disorders. American Journal of Psychiatry. 1984; 141:684-6. [PubMed: 6538762]

Schmidt PJ, Nieman LK, Danaceau MA, Adams LF, Rubinow DR. Differential behavioral effects of gonadal steroids in women with and in those without premenstrual syndrome. New England Journal of Medicine. 1998; 338:209-16. [PubMed: 9435325]

Schmidt PJ, Nieman LK, Grover GN, Muller KL, Merriam GR, Rubinow DR. Lack of effect of induced menses on symptoms in women with premenstrual syndrome. New England Journal of Medicine. 1991; 324:1174-9. [PubMed: 2011161]

Schnurr PP. Some correlates of prospectively defined premenstrual syndrome. American Journal of Psychiatry. 1988; 145:491-4. [PubMed: 3348453]

Schnurr PP. Measuring amount of symptom change in the diagnosis of premenstrual syndrome. Psychological Assessment. 1989; 1:277-83.

Schoukens, J.; Pintelon, R. Identification of linear systems: a practical guideline for accurate modeling. London: Pergamon Press; 1991. p. 151

Smith MJ, Schmidt PJ, Rubinow DR. Operationalizing DSM-IV criteria for PMDD: selecting symptomatic and asymptomatic cycles for research. Journal of Psychiatric Research. 2003; 37:7583. [PubMed: 12482472]

Spitzer, RL.; Williams, JB.; Gibbon, M.; First, MB. Structured clinical interview for DSM-III-R. New York, NY: Biometrics Research Department, New York State Psychiatric Institute; 1990. patient edition

Steiner M, Haskett RF, Carroll BJ. Premenstrual tension syndrome: the development of research diagnostic criteria and new rating scales. Acta Psychiatrica Scandinavica. 1980; 62:177-90. [PubMed: 7193399]

Sternfeld B, Swindle R, Chawla A, Long S, Kennedy S. Severity of premenstrual symptoms in a health maintenance organization population. Obstetrics and Gynecology. 2002; 99:1014-24. [PubMed: 12052592]

Veldhuis JD, Keenan DM, Pincus SM. Motivations and methods for analyzing pulsatile hormone secretion. Endocrine Reviews. 2008; 29:823-64. [PubMed: 18940916]

Yeragani VK, Pohl R, Mallavarapu M, Balon R. Approximate entropy of symptoms of mood: an effective technique to quantify regularity of mood. Bipolar Disorders. 2003; 5:279-86. [PubMed: 12895205]

Yeragani VK, Radhakrishna RK, Tancer M, Uhde T. Nonlinear measures of respiration: respiratory irregularity and increased chaos of respiration in patients with panic disorder. Neuropsychobiology. 2002; 46:111-20. [PubMed: 12422057]

Yonkers KA, Brown C, Pearlstein TB, Foegh M, Sampson-Landers C, Rapkin A. Efficacy of a new low-dose oral contraceptive with drospirenone in premenstrual dysphoric disorder. Obstetrics and Gynecology. 2005; 106:492-501. [PubMed: 16135578]

Yonkers KA, Halbreich U, Freeman E, Brown C, Endicott J, Frank E, et al. Symptomatic improvement of premenstrual dysphoric disorder with sertraline treatment: a randomized controlled trial. Journal of the American Medical Association. 1997; 278:983-8. [PubMed: 9307345] 

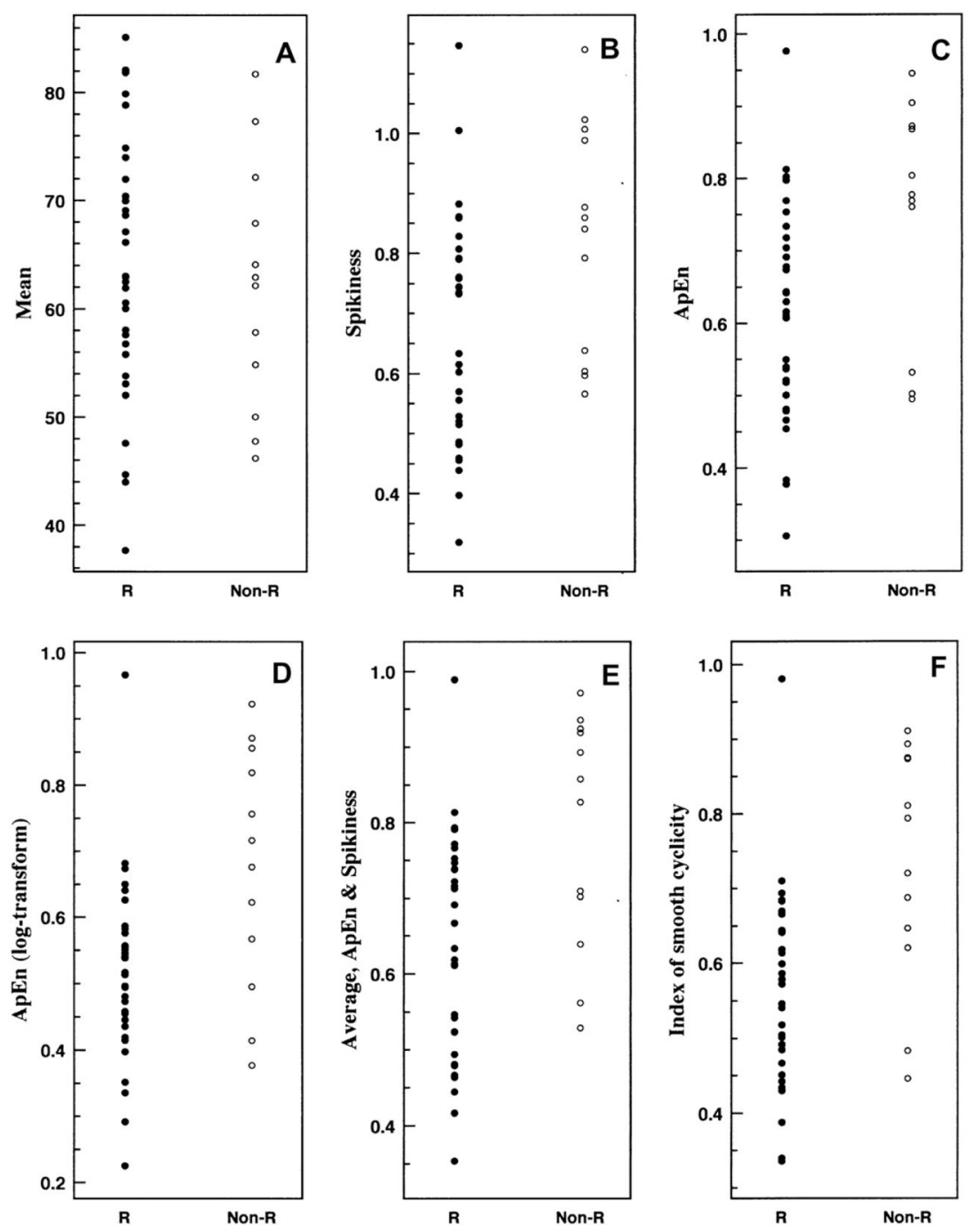

Fig. 1.

Individual subject values of responders and nonresponders for each of (A) mean; (B) Spikiness; (C) ApEn; (D) ApEn of the log-transformed series; (E) normalized average of ApEn and Spikiness; (F) index of smooth cyclicity (normalized average of ApEn of the logtransformed series and Spikiness). R indicates responder, Non-R indicates nonresponder. Each subject value is averaged over the three symptoms of the visual-analogue scale (VAS): sadness, anxiety, and irritability. 

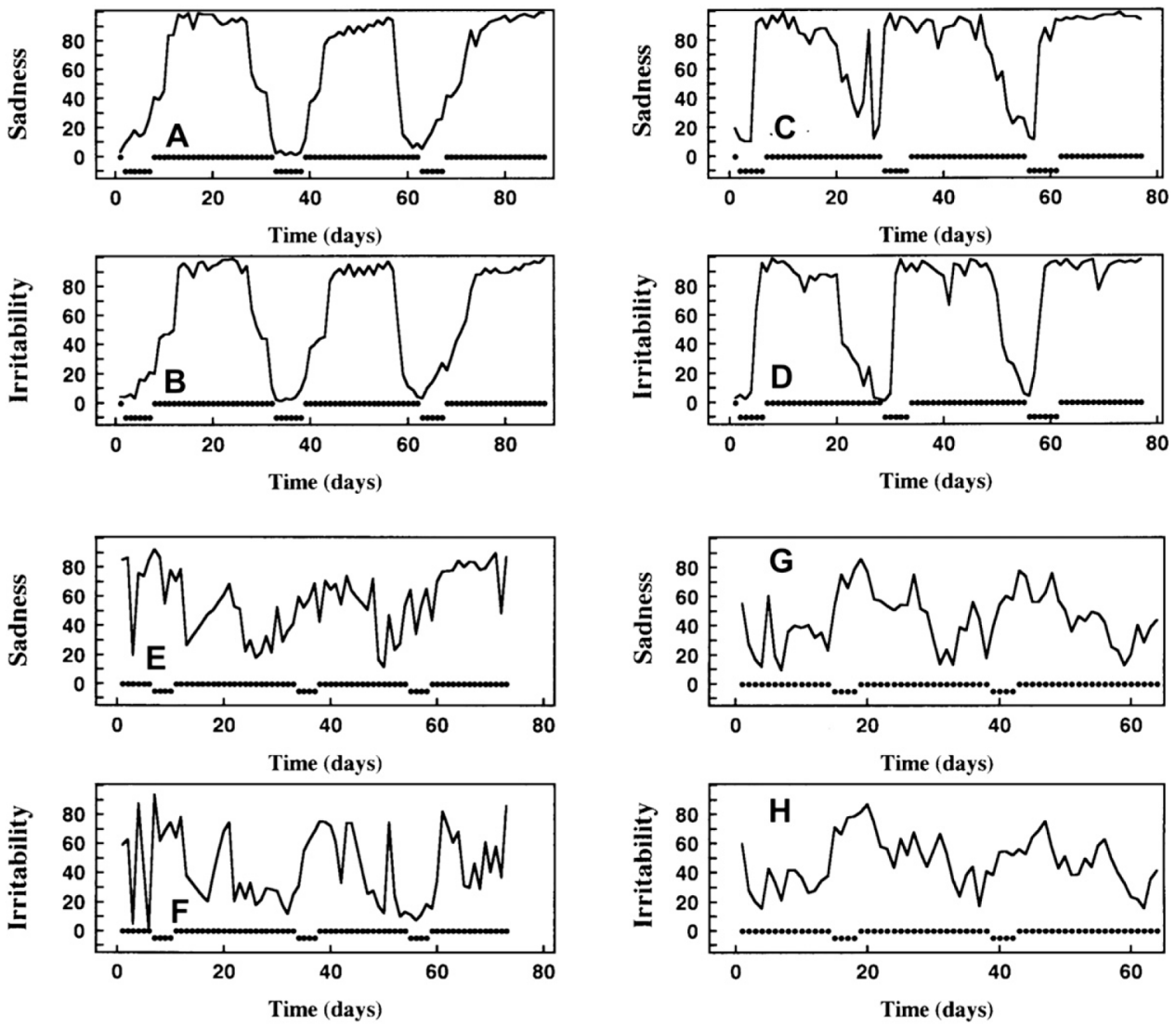

Fig. 2.

Representative pre-trial daily mood rating profiles for two responders and two nonresponders. VAS sadness and irritability series for responder \#1 are upper left, panels (A, B); for responder \#2, upper right, panels (C, D); for nonresponder \#1, lower left, (E, F); for nonresponder \#2, lower right, $(\mathrm{G}, \mathrm{H})$. Timing of menses are indicated by dips in the dotted marks displayed at the 0 level of each panel. Average values across all symptoms for each statistic are: responder \#1, mean, 60.58; Spikiness, 0.320; ApEn, 0.378; logtransformed ApEn, 0.352; normalized average of ApEn and Spikiness, 0.354; Index of smooth cyclicity, 0.340; responder \#2; mean, 72.00; Spikiness, 0.557; ApEn, 0.501; logtransformed ApEn, 0.292; normalized average of ApEn and Spikiness, 0.524; Index of smooth cyclicity, 0.388; nonresponder \#1; mean, 50.06; Spikiness, 1.024; ApEn, 0.873; logtransformed ApEn, 0.820; normalized average of ApEn and Spikiness, 0.936; index of smooth cyclicity, 0.894; nonresponder \#2; mean, 46.21; Spikiness, 0.793; ApEn, 0.905; logtransformed ApEn, 0.923; normalized average of ApEn and Spikiness, 0.858; Index of smooth cyclicity, 0.876 . On visual inspection, observe 'classic' cyclic dynamics for each symptom for the two responders in (A, B) and (C, D). For each nonresponder, the mood ratings sets $(\mathrm{E}, \mathrm{F})$ and $(\mathrm{G}, \mathrm{H})$ satisfy the quantitative criteria for PMDD. Yet note dynamic inconsistency across months and between symptoms, greater irregularity, and greater Spikiness, typically manifesting numerous brief moderate-to-large drops in mood or depressive epochs not proximate to menses. 
Table 1

Demographic and baseline characteristics.

\begin{tabular}{lcc}
\hline Characteristics & Responders $(\boldsymbol{n}=\mathbf{3 3})$ & Nonresponders $(\boldsymbol{n}=\mathbf{1 2})$ \\
\hline Age (y) & $38.8 \pm 6.5$ & $40.8 \pm 5.3$ \\
BMI $\left(\mathrm{kg} / \mathrm{cm}^{2}\right)$ & $25.5 \pm 4.8$ & $26.6 \pm 4.8$ \\
No. Married (\%) & $16(48.5)$ & $4(33.3)$ \\
No. with children (\%) & $22(66.7)$ & $6(50.0)$ \\
No. with past major depression (\%) & $10(30.3)$ & $5(41.7)$ \\
No. with symptom exacerbation (flare) after first injection of leuprolide (\%) ${ }^{a}$ & $16(48.5)$ & $4(33.3)$ \\
Severity of hot flushes ${ }^{b}$ & $2.7 \pm 1.2$ & $2.3 \pm 0.8$ \\
Plasma hormone levels ${ }^{c}$ & & \\
Progesterone [ng/ml] & & $0.3 \pm 0.1$ \\
Estradiol [pg/ml] & $0.3 \pm 0.2$ & $20.2 \pm 12.7$ \\
LH [IU/L] & $20.9 \pm 10.4$ & $3.0 \pm 2.8$ \\
FSH [IU/L] & $1.6 \pm 1.6$ & $7.6 \pm 4.0$ \\
\hline
\end{tabular}

Statistical values given as mean $\pm \mathrm{SD}$ for numerical variables and $n(\%)$ for categorical variables.

All comparisons between Responders and Nonresponders showed no statistically significant results, $P>0.05$ for all comparisons.

${ }^{a}$ A clinically significant symptom exacerbation was defined by the occurrence of a rating below a threshold level for two or more consecutive days during the first month of leuprolide, for any symptom. The threshold level (for each symptom) was defined as the value obtained by subtracting $30 \%$ of the range of VAS from the baseline mean of the particular symptom.

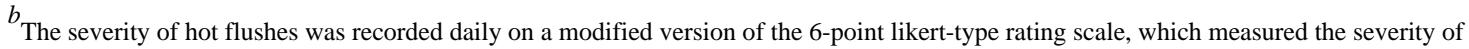
symptoms in which $1=$ no symptoms, $6=$ extremely severe symptoms. Values represent daily ratings during the last two weeks on leuprolide.

${ }^{c}$ Values represent hormone levels during the last two months on leuprolide. 
